\title{
Möglichkeiten zur Optimierung eines bestehenden Grundwassermodells zur besseren Beurteilung der Wasserqualität bei der Untersuchung der chemischen Stabilität von Uferfiltrat
}

\author{
Sebastian Handl · Ernest Mayr · Reinhard Perfler
}

Online publiziert: 24. Juli 2019

(c) Der/die Autor(en) 2019

Zusammenfassung Bei der Untersuchung der chemischen Stabilität des Wassers während der Uferfiltration (auf Basis von Wasserqualitätsdaten) spielt die Aufenthaltszeit des Wassers von der Infiltration in den Grundwasserbegleitstrom bis zum Erreichen des Brunnens eine wichtige Rolle. Die Fließgeschwindigkeiten in Flüssen sind naturgemäß um einige Skalen höher als jene des Grundwassers, welches dem Brunnen zuströmt. Direkte Vergleiche zwischen Analyseergebnissen aus dem Oberflächengewässer mit Analyseergebnissen aus dem Brunnen, welche zum selben Zeitpunkt genommen wurden, sind nicht aussagekräftig, da Anteile aus der aktuellen Flussprobe erst wesentlich später die Messstelle im Grundwasser erreichen. In der vorliegenden Untersuchung werden die Herausforderungen und Lösungsmöglichkeiten bei der Optimierung der numerischen Grundwassermodellierung bei einem Uferfiltratsstandort dargestellt. Ausgehend von einem bestehenden Modell wird eine Adaption vorgenommen, um die hydrogeologischen Verhältnisse besser darzustellen. Dazu wird die Methodik zur Füllung von Messlücken in Grundwassersonden, welche als Randbedingung in das Modell eingehen, dargestellt. Die hydrogeologischen Parameter des Modells werden anhand zweier Pumpversuche in zwei Szenarien kalibriert und die Güte der Modellanpassung untersucht. Auf Basis des kalibrierten

DI S. Handl (凶) • DI E. Mayr •

PD DI Dr. R. Perfler

Department

Wasser-Atmosphäre-Umwelt,

Institut für Siedlungswasserbau,

Industriewasserwirtschaft und

Gewässerschutz, Universität

für Bodenkultur Wien,

Muthgasse 18, 1190 Wien, Österreich

sebastian.handl@boku.ac.at
Modells wird für jede Probenahme aus dem Brunnen eine Verteilung der Aufenthaltszeit auf Basis des advektiven Stofftransports mittels Partikeltracking ermittelt. Auf dieser Basis kann der Abgleich von Proben aus dem Oberflächengewässer mit Proben aus dem Grundwasser erfolgen und die Reinigungsleistung ermittelt werden.

\section{Schlüsselwörter}

Grundwassermodellierung .

Uferfiltration - Aufenthaltszeit .

Untergrundpassage

Possibilities in the optimisation of an existing groundwater model to enhance the assessment of water quality for investigations of the chemical stability of riverbank filtrate

Abstract The residence time of water from infiltration through the riverbed until it reaches the well plays an important role for the investigation of water quality alteration during riverbank filtration. The flow velocities in rivers are naturally of higher magnitude compared to groundwater flow velocities. Comparisons of measurements for water quality parameters between samples from the river and samples from wells have to take this into account. The presented study shows challenges and possible solutions in modelling groundwater flow at riverbank filtration sites. It covers the adaption of an existing groundwater model from an earlier investigation to better represent the hydrogeological processes. The hydrogeological parameters of the model are calibrated with the data of two pumping tests. The calibrated model is used to calculate the distribution of residence time for each sampling time in the well via reverse particle tracking. This results built the basis for the align- ment of well with river samples in order to investigate water quality alterations during riverbank filtration.

Keywords Groundwater modelling . Riverbank filtration - Residence time . Underground passage

\section{Einleitung}

Die Beurteilung der Stabilität der chemischen Wasserqualität wurde im Rahmen eines gemeinsamen Projektes mit dem Institut für Analytische Chemie (BOKU-IAC) durchgeführt. Im Zuge dieser Arbeiten war es notwendig, einen Abgleich zwischen Analyseergebnissen aus dem Grundwasser mit Analyseergebnissen aus dem Oberflächengewässer herzustellen, um die Ausgangswasserqualität für die entnommenen Grundwasserproben darstellen zu können. Zu diesem Zweck wurden die hier dargestellten Methoden entwickelt und angewandt.

\section{Aufbau des Grundwassermodells}

\subsection{Grundlage und Adaption}

Das bestehende Grundwassermodell führt in seinem Aufbau mit inaktiven Zellen an den Modellgrenzen normal zum Oberflächengewässer auch unterhalb des Flusses zu einer Negierung des Grundwasserbegleitstroms. Der Grundwasserbegleitstrom bezeichnet die Strömung von Grundwasser entlang von Flüssen. Im Zuge der Wiederaufnahme des Grundwassermodells zur Berechnung der Wasserbewegung wurde auch in den Zellen unterhalb des Oberflächengewässers an den Modellgrenzen ein - über die Zeit variabler - Wasserstand als Randbedingung gesetzt. Im oberen Layer des Modells wirkt die „Fluss“-Randbedingung (River-Paket). Für alle Zellen, welche eine Randbedin- 

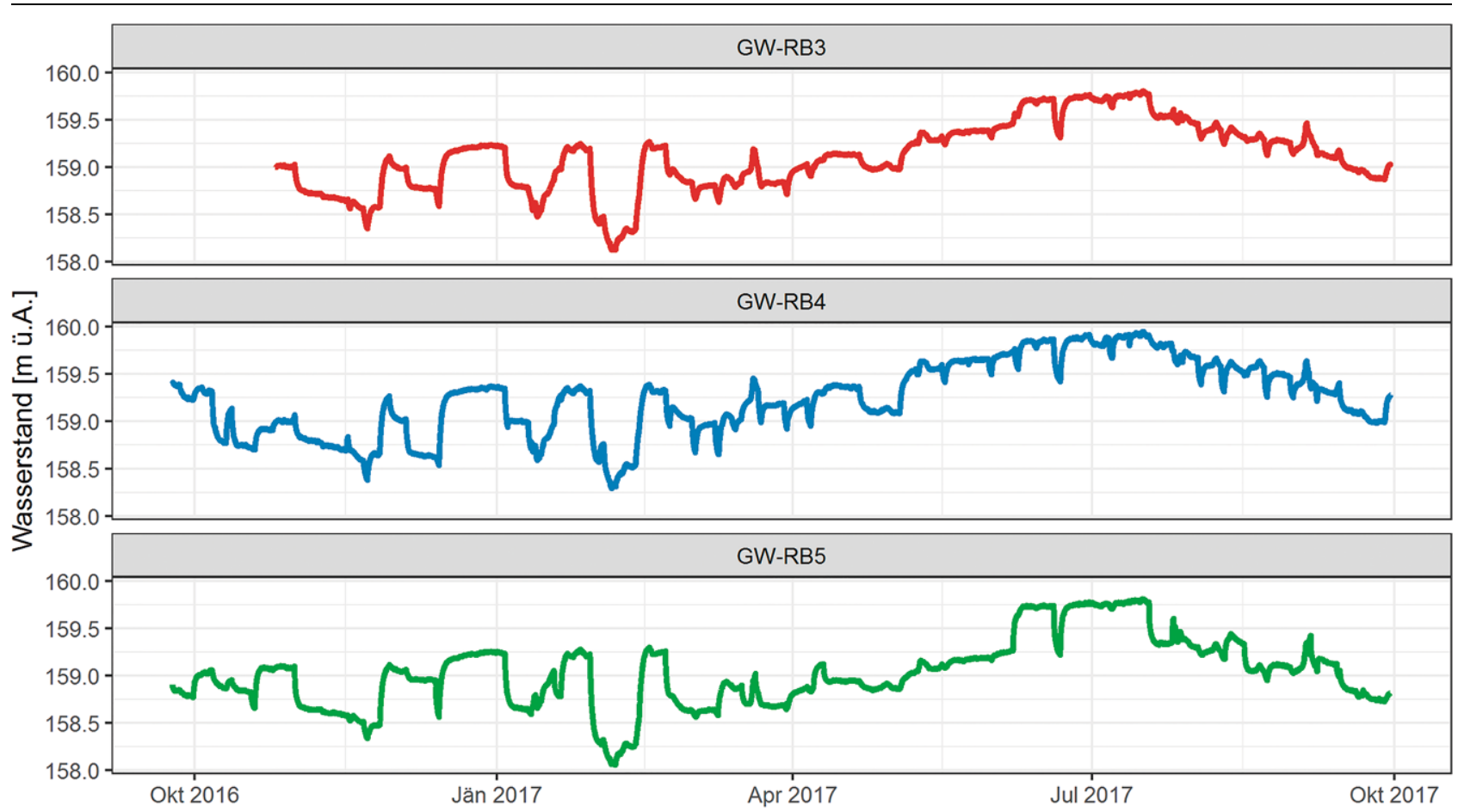

Grundwassersonde - GW-RB3 - GW-RB4 - GW-RB5

Abb. 1 Zeitreihen der Wasserstände der Sonden GW-RB3 bis GW-RB5

gung darstellen und die zwischen den Grundwasser-Messstellen liegen, werden die jeweiligen Wasserstände der nächstgelegenen Grundwassersonden linear interpoliert.

Die Anpassung der zeitlichen Diskretisierung des Grundwassermodells an den neuen Untersuchungszeitraum erfolgte mit der Software PMWIN (Chiang und Kinzelbach 2001). Die Eingabe der Werte für alle Parameter des Grundwassermodells in jeder Zelle (Durchlässigkeitsbeiwerte, Startwasserstände, zeitlicher Verlauf der Wasserstände in den Randsonden etc.) erfolgte durch Anpassung der ursprünglichen Eingangsdateien unter Verwendung der frei verfügbaren Statistiksoftware R (R Core Team 2018).

\subsection{Füllung von Lücken in den} Messreihen der Randbedingungen

\subsubsection{Datengrundlage Randbedingungen}

Die Wasserstände von 10 Grundwassermessstellen gehen als Grundlage zur Berechnung der Randbedingungen in das Grundwassermodell ein. Bei der Messstelle GW-RB3 kam es zu Beginn des Untersuchungszeitraums zu

Tab. 1 Anpassungsparameter und Regressionskoeffizienten des linearen Regressionsmodells der Messstelle GW-RB3

\begin{tabular}{|c|c|c|c|c|c|c|c|}
\hline Modell & $p$-Wert & $\mathrm{R}^{2}$ & a & b & Sonde-B & c & Sonde-C \\
\hline GW-RB3 & $<2,2 * 10^{-16}$ & 0,990 & 6,443 & 0,416 & GW-RB5 & 0,543 & GW-RB4 \\
\hline
\end{tabular}

einem kurzzeitigen Ausfall des Wasserstandsloggers. Diese Messstelle liegt jedoch räumlich zwischen den Messstellen GW-RB4 und GW-RB5. Abb. 1 gibt die Zeitreihen der drei Grundwassermessstellen wieder. Im Zeitraum, in welchem für alle Sonden Messwerte vorliegen (ab zirka November 2016), ist ein starker Zusammenhang zwischen den verschiedenen Grundwasserständen erkennbar. Auf Basis dieses Bereichs wurden ein lineares Regressionsmodell entsprechend der Gl. 1 für die Messstelle GW-RB3 angepasst und die Koeffizienten der Regression ( $a, b$ und c) geschätzt. Tab. 1 gibt die Ergebnisse der Anpassungen wieder. Der $p$-Wert stellt dabei das Signifikanzniveau des Regressionsmodells für die geschätzte Größe (Wasserstand in der gesuchten Messstelle) dar. Liegt der $p$-Wert unter 0,05 , ist das Modell für die geschätzte Größe signifikant. Für das vorliegende Modell liegt der $p$-Wert $<2,2 * 10^{-16}$. Dies bedeutet, dass das Modell hoch signifikant für die gesuchte Größe ist.
Das Bestimmtheitsmaß $\left(R^{2}\right)$ gibt an, wie groß der Anteil der gesamten Varianz der geschätzten Größe durch das Modell erklärt werden kann und liegt daher zwischen 0 (0\%) und 1 (100\%). Es werden $99 \%$ der Varianz durch das Modell erklärt. Dies zeigt einen sehr hohen Zusammenhang zwischen den gemessenen und den durch das Modell geschätzten Werten.

$$
\begin{aligned}
W S T_{G W-R B 3}= & a+b \cdot W S T_{G W-R B 5} \\
& +c \cdot W S T_{G W-R B 4}
\end{aligned}
$$

$W S T_{G W-R B 3} \quad$ Wasserstand der gesuchten Messstelle GW-RB3 [m ü. A.]

$W_{S T} T_{G W-R B 5}$ Wasserstand der GWRB5 [m ü. A.]

$W S T_{G W-R B 4}$ Wasserstand der GWRB4 [m ü. A.]

$a, b, c \quad$ Koeffizienten der Regression 


\section{Originalarbeit}

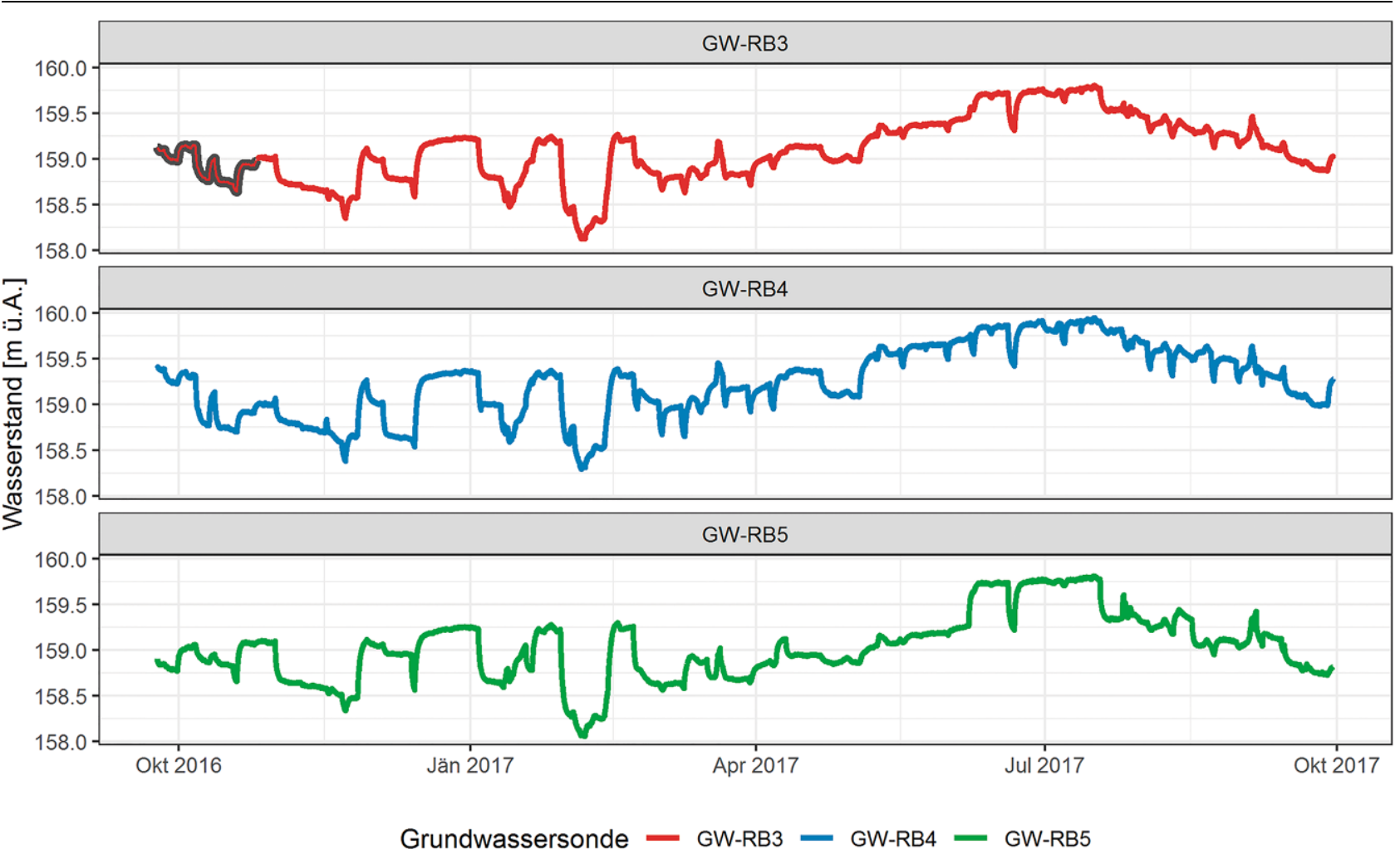

Abb. 2 Wasserstände der Messstellen GW-RB3 bis GW-RB5 (Lücken in der Messreihe gefüllt)

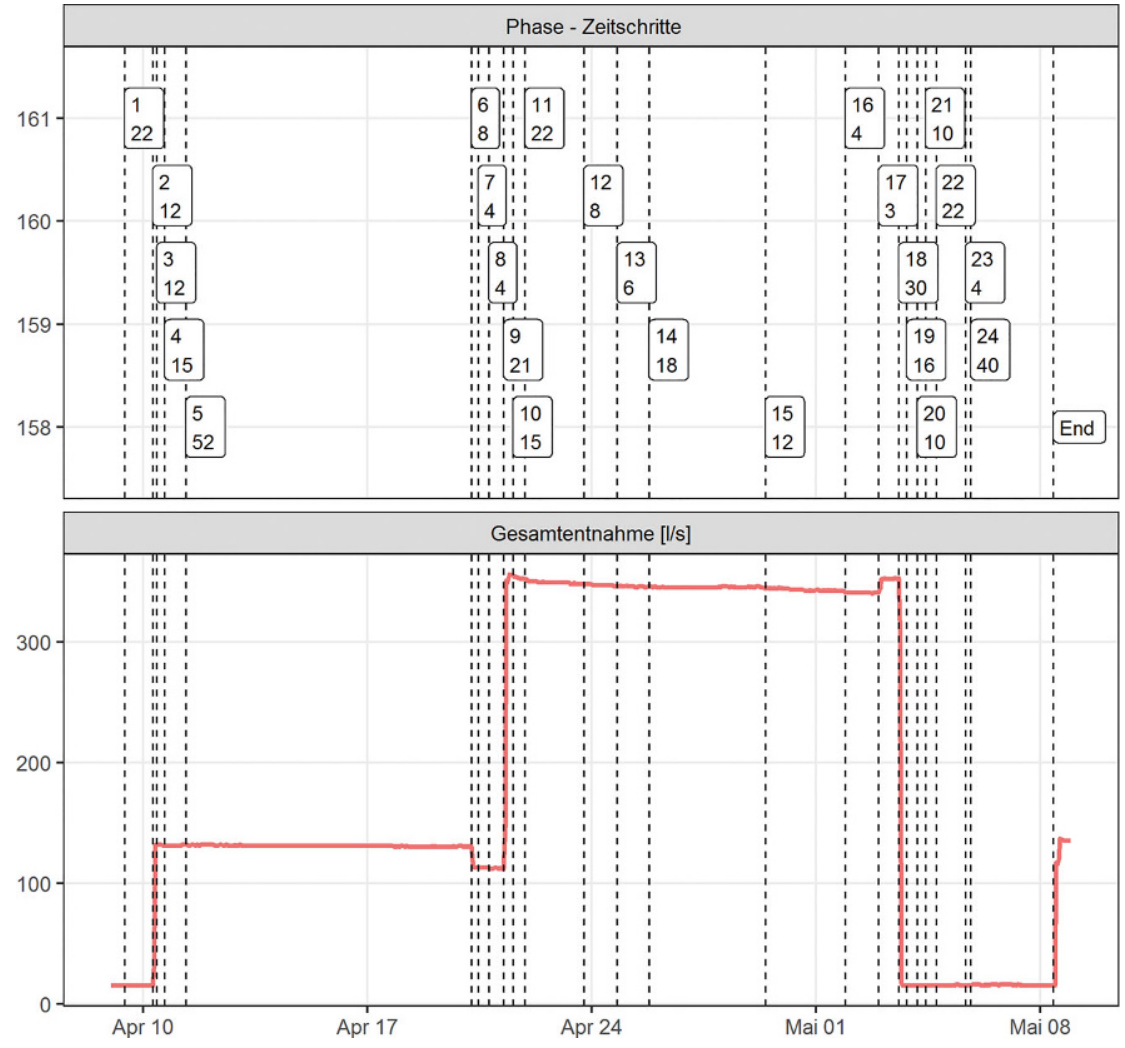

Abb. 3 Zeitraum der transienten Modellierung (Pumpversuch2): Phasen, Zeitschritte, Gesamtentnahme

\subsubsection{Anwendung der Regressionsmodelle}

Abb. 2 zeigt die Zeitreihen der Wasserstände der Messstellen GW-RB3 bis GW-RB5. Die dunkelgrau hinterlegten Bereiche kennzeichnen den Abschnitt, welcher mittels des linearen Regressionsmodells berechnet wurde.

\subsection{Kalibrierung}

\subsubsection{Hydraulische Eigenschaften}

Zur Kalibrierung des Grundwassermodells müssen drei Arten von Parametern angepasst werden. Die ersten beiden betreffen den Untergrund selbst, also die Eigenschaften des Grundwasserkörpers. Der hydraulische Durchlässigkeitsbeiwert nach Darcy ( $\mathrm{k}_{\mathrm{f}}$-Wert) gibt an, wie viel Wasser $\left(\mathrm{m}^{3} / \mathrm{s}\right)$ bei einem Wasserstandgefälle (hydraulischer Gradient) von 1 durch eine $1 \mathrm{~m}^{2}$ große Fläche strömt. Die effektive Porosität ( $\left.\mathrm{n}_{\text {eff }}\right)$ gibt den Anteil des für die Strömung des Wassers zur Verfügung stehenden Volumens am Gesamtvolumen des Untergrunds an. Im vorliegenden Fall eines ungespannten Grundwasserkörpers kann daraus gleichzeitig die durch eine Wasserstandabsenkung frei werdende 


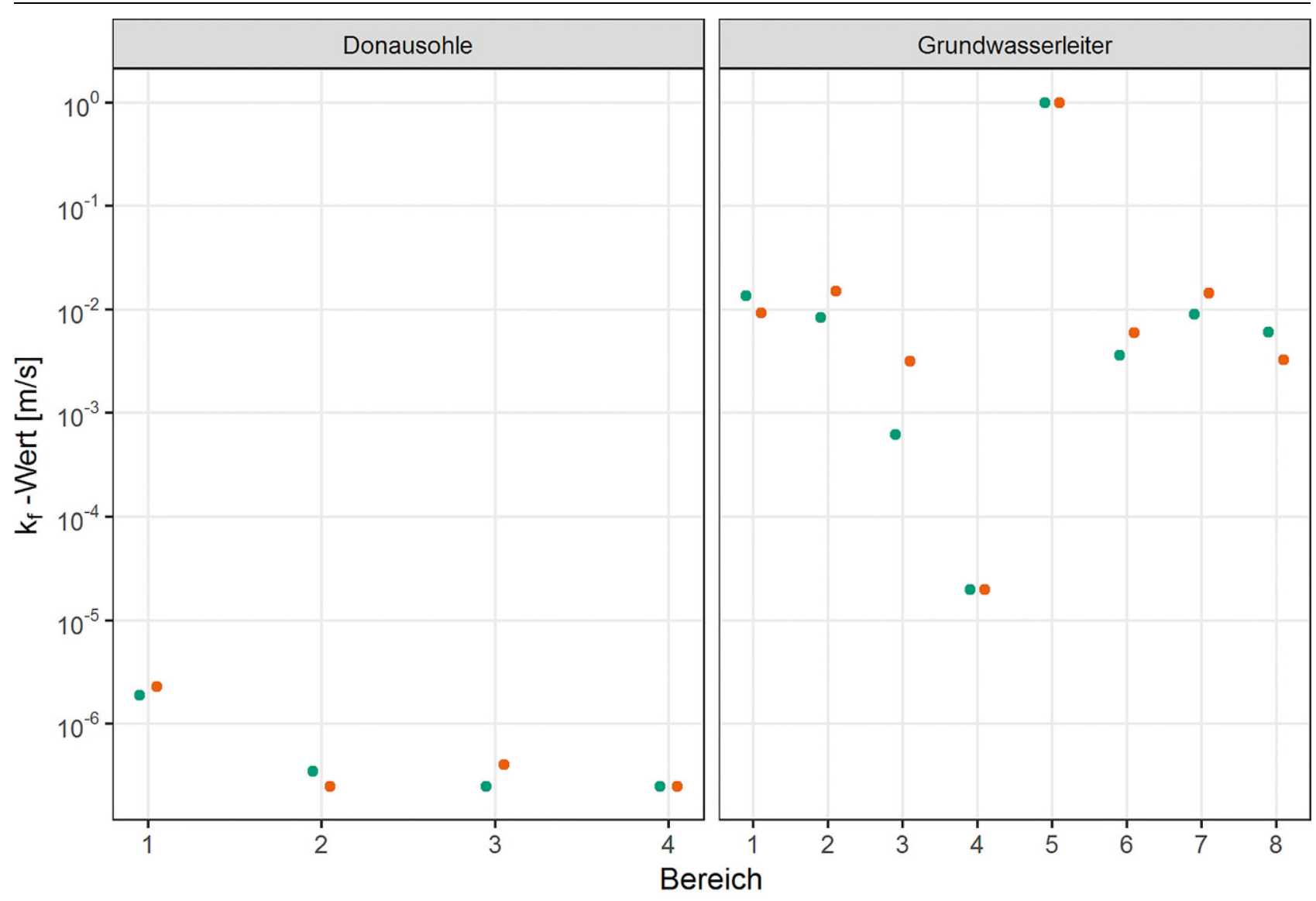

Kalibrierungsvariante $1 \bigcirc$ Kalibrierungsvariante 2

Abb. 4 Ergebnisse der Parameteroptimierung der $\mathrm{k}_{\mathrm{f}}$-Werte

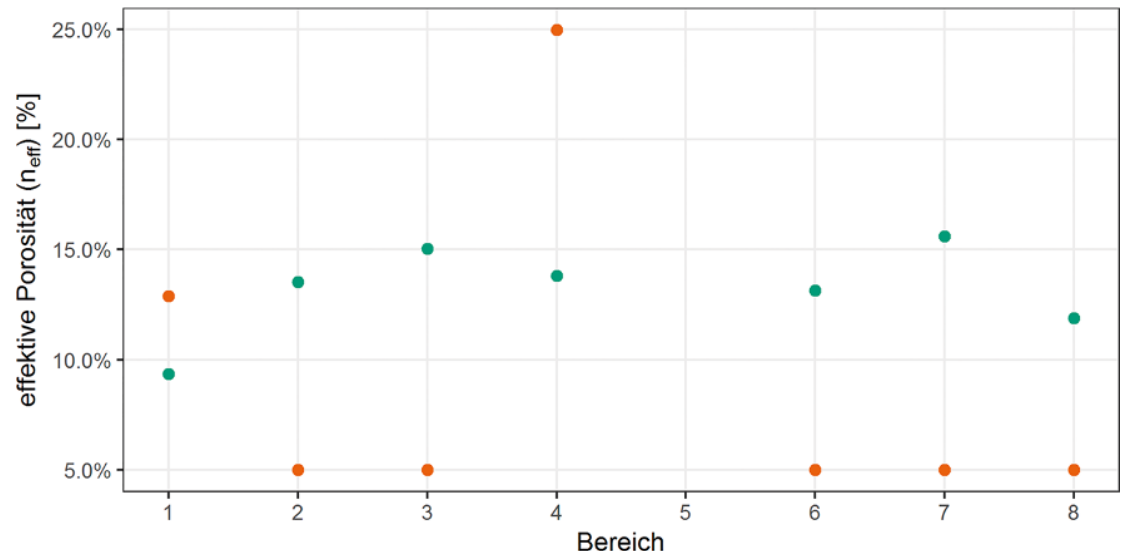

- Kalibrierungsvariante 1 Kalibrierungsvariante 2

Abb. 5 Ergebnisse der Parameteroptimierung der $\mathrm{k}_{\mathrm{f}}$-Werte

Wassermenge berechnet werden. Die Durchlässigkeit des Flussbetts stellt die dritte Art von Parametern dar. Sie entspricht dem $\mathrm{k}_{\mathrm{f}}$-Wert (siehe oben) der Sohle des Oberflächengewässers.

\subsubsection{Räumliche Zonierung}

Die Eigenschaften von Grundwasserkörper und Flussbett sind auch räumlich variabel. Bereiche mit homogenen Eigenschaften wurden aus dem zugrun- de liegenden Modell übernommen. Der Grundwasserkörper im Modell wurde in 8 Bereiche unterteilt, für welche wiederum homogene Eigenschaften $\left(\mathrm{k}_{\mathrm{f}}\right.$-Wert und $\mathrm{n}_{\text {eff }}$ ) angenommen wurden. Die Infiltrationszone des Oberflächengewässers im Modell wurde in vier Bereiche eingeteilt. Für diese werden homogene Eigenschaften der Flusssohle ( $\mathrm{k}_{\mathrm{f}}$-Werte) angenommen.

\subsubsection{Ermittlung der Parameter}

Aus der Kombination der drei Arten von Parametern und der Zonierung nach homogenen Bereichen ergeben sich für das Grundwassermodell in Summe 20 Parameter, welche angepasst werden, damit das Modell auch die tatsächlichen Verhältnisse im Untersuchungsgebiet widerspiegelt. Bei der inversen Modellanwendung wird das Grundwassermodell anhand von beobachteten Messwerten im Untersuchungsgebiet aufgebaut. Die Wasserstände in den Randsonden und die 


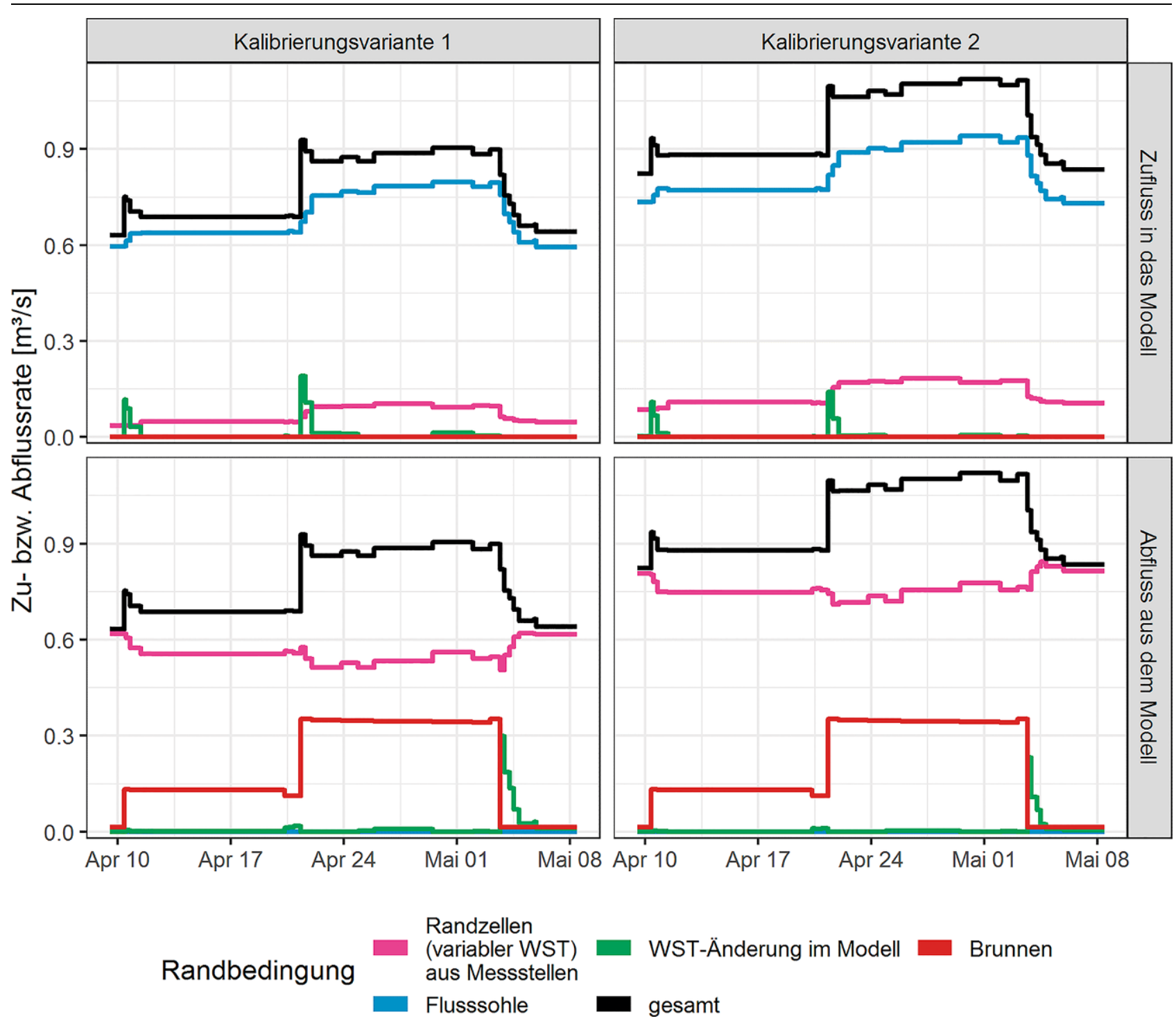

Abb. 6 Wassermengenbudget nach Art der Modellgrenze und Kalibrierungsvariante

Entnahmemengen aus den Brunnen werden als Randbedingungen herangezogen. Auf Grundlage der Wasserstandmessungen weiterer Grundwassersonden im Untersuchungsgebiet, werden in weiterer Folge die hydraulischen Parameter schrittweise so lange angepasst, bis eine möglichst gute Übereinstimmung zwischen den berechneten Wasserständen im Modell und den beobachteten im Untersuchungsgebiet gegeben ist. Zur Schätzung der Parameter wurde das PEST-Software-Paket verwendet, welches in der Software PMWIN (Chiang und Kinzelbach 2001) integriert ist. Die Optimierung erfolgt in zwei Schritten. Im ersten Schritt werden die $\mathrm{k}_{\mathrm{f}}$-Werte des Grundwasserkörpers und der Flusssohle anhand mehrerer stationärer Zustände angepasst. Im zweiten Schritt werden die Parameter aus dem ersten Schritt übernommen und der Kalibrierungszeitraum wird transient modelliert, um die effektive Porosität der homogenen Bereiche im Grundwasserkörper zu optimieren.

Kalibrierungsschritt 1: Durchlässigkeitsbeiwerte Es wurden zwei unterschiedliche Zeiträume zur Kalibrierung der $\mathrm{k}_{\mathrm{f}}$-Werte des Modells herangezogen. Dadurch werden zwei verschiedene Parametersets erzeugt und ermöglichen damit Aussagen über die Bandbreite in Bezug auf die zu berechnenden Größen (Aufenthaltszeit).

In jeder Phase der Modelle werden die Randbedingungen (Wasserstände in den Randzellen und im Oberflächengewässer und Entnahmemengen aus den Brunnen) entsprechend der Verhält- nisse zum stationären Endzeitpunkt der jeweiligen Phase angesetzt. Unter Anwendung des PEST-Pakets werden die Parameter für die Durchlässigkeitsbeiwerte des Grundwasserleiters und jene der Gewässersohle optimiert. Die effektive Porosität hat in diesem Modellaufbau keinen Einfluss auf die Optimierung.

Kalibrierungsschritt 2: effektive Porosität Die Ermittlung der Parameter der effektiven Porosität in den 8 homogen angenommenen Zonen der Grundwasserleiters erfolgt durch transiente Modellierung des Pumpversuchs 2. Die Durchlässigkeitsbeiwerte werden dabei aus dem ersten Kalibrierungsschritt übernommen. Abb. 3 zeigt für den Modellzeitraum im oberen Teil die Unterteilung in Phasen (1 bis 24) sowie 


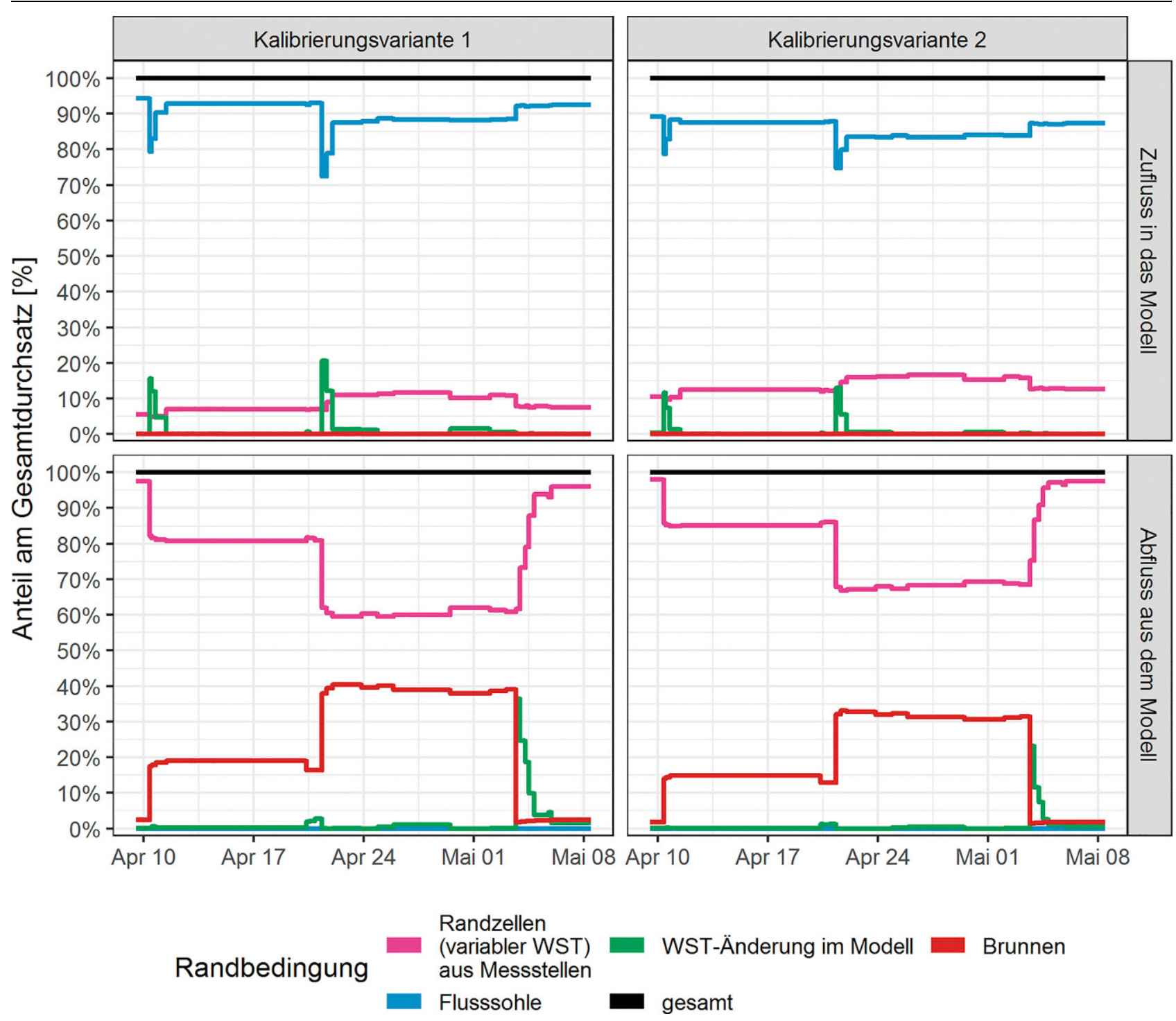

Abb. 7 Wassermengenbudget nach Art der Modellgrenze und Kalibrierungsvariante - relative Anteile am Gesamtdurchsatz

die Anzahl der Zeitschritte innerhalb der Phasen. Im unteren Bereich wird der Verlauf der Gesamtentnahme in $1 / \mathrm{s}$ dargestellt.

Gegenüberstellung der Kalibrierungsvarianten Die Ergebnisse aus der Parameteroptimierung der Durchlässigkeitsbeiwerte sind in Abb. 4 dargestellt. Für beide Kalibrierungsvarianten liegen die geschätzten Werte der inversen Modellierung in ähnlichen Skalenbereichen. Die Nummerierung der Bereiche entspricht den homogenen Bereichen, wie weiter oben dargestellt. Für den Bereich 4 der $\mathrm{k}_{\mathrm{f}}$-Werte im Grundwasserleiter ergibt sich in beiden Kalibrierungsvarianten der geringste zulässige Wert $\left(2 * 10^{-5} \mathrm{~m} / \mathrm{s}\right)$. Der Bereich 5 der $\mathrm{k}_{\mathrm{f}}$-Werte entspricht den Zellen im Nahbereich der Brunnen. Dieser Bereich wurde zum Zweck der numerischen Stabilität des Modells mit $1 \mathrm{~m} / \mathrm{s}$ festgelegt und nicht bei der Parameteroptimierung berücksichtigt. Die Durchlässigkeiten der Gewässersohle können aus den Optimierungen nicht direkt abgeleitet werden. Den dargestellten $\mathrm{k}_{\mathrm{f}^{-}}$ Werten der Gewässersohle liegt die Annahme einer $20 \mathrm{~cm}$ dicken Sohlschicht zugrunde. Sie liegen naturgemäß deutlich geringer als die Durchlässigkeiten im Grundwasserkörper selbst.

Abb. 5 zeigt die Ergebnisse der Parameteroptimierung in Bezug auf die effektive Porosität. In der Kalibrierungsvariante 1 liegen alle Werte in ähnlichen Bereichen. Bei der Kalibrierungsvariante 2 fällt auf, dass nur der Bereich 1 etwa den Werten der Kalibrierungsvariante 2 entspricht und für alle anderen Berei- che ein sehr niedriger $(5 \%)$ oder relativ hoher $(25 \%)$ Wert ermittelt wurde.

In Abb. 6 werden die Verläufe der $\mathrm{Zu}$ - (oberer Diagrammbereich) und Abflüsse (unterer Diagrammbereich) über den Pumpversuch 2 gesondert nach Art der Modellgrenze für beide Kalibrierungsvarianten dargestellt. Generell herrscht in der Kalibrierungsvariante 2 ein wesentlich höherer Wassermengengesamtdurchsatz (schwarze Linie) vor. Bezogen auf die Variante 1 wird das Modell mit den Parametern aus Variante 2 von zwischen 18 und $30 \%$ (im Mittel ca. 25\%) mehr Wasser je Zeiteinheit durchströmt. Die Durchsatzraten über die Brunnen (rote Linie) sind naturgemäß in beiden Varianten gleich. In beiden Varianten wird die Entnahme aus den Brunnen (rote Li- 


\section{Originalarbeit}

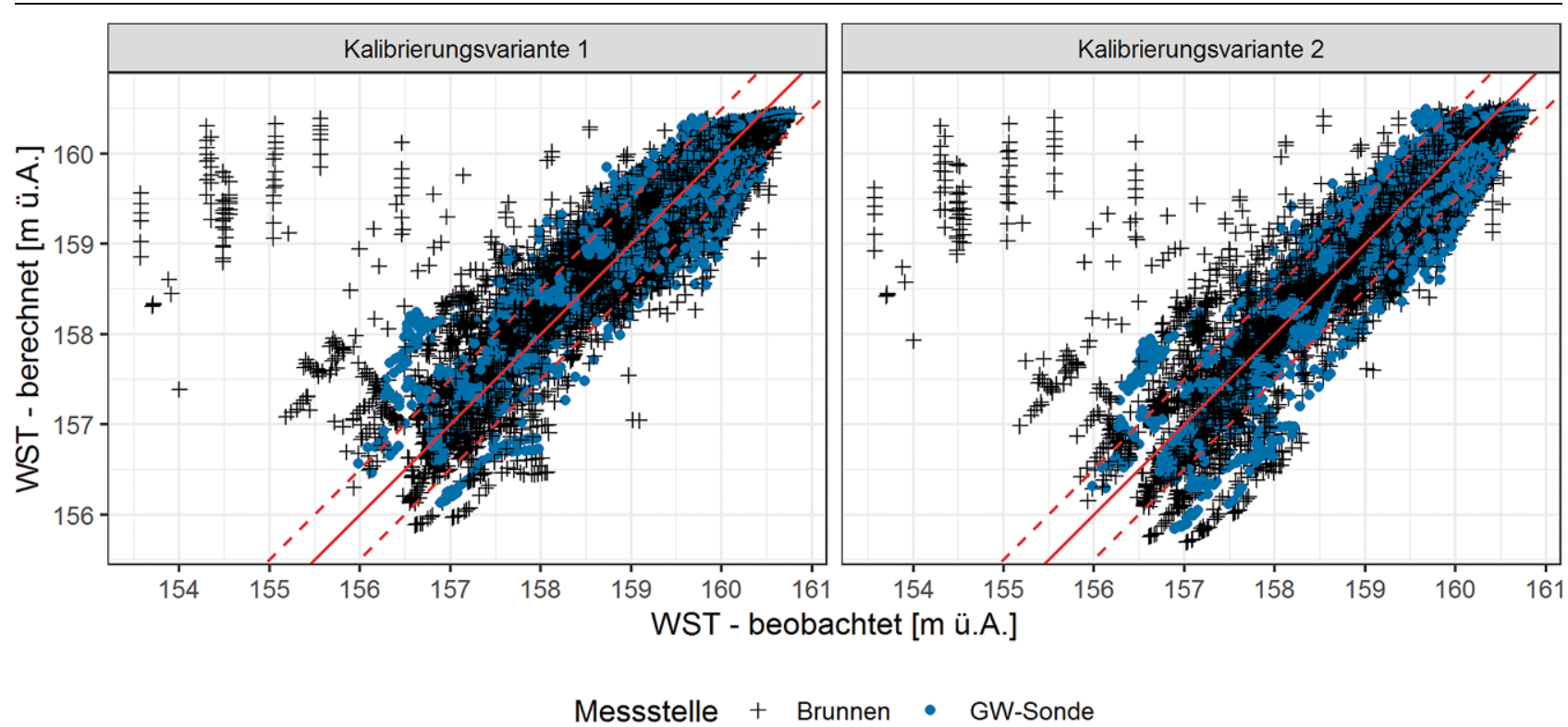

Abb. 8 Gegenüberstellung simulierter und beobachteter Wasserstände nach Kalibrierungsvarianten
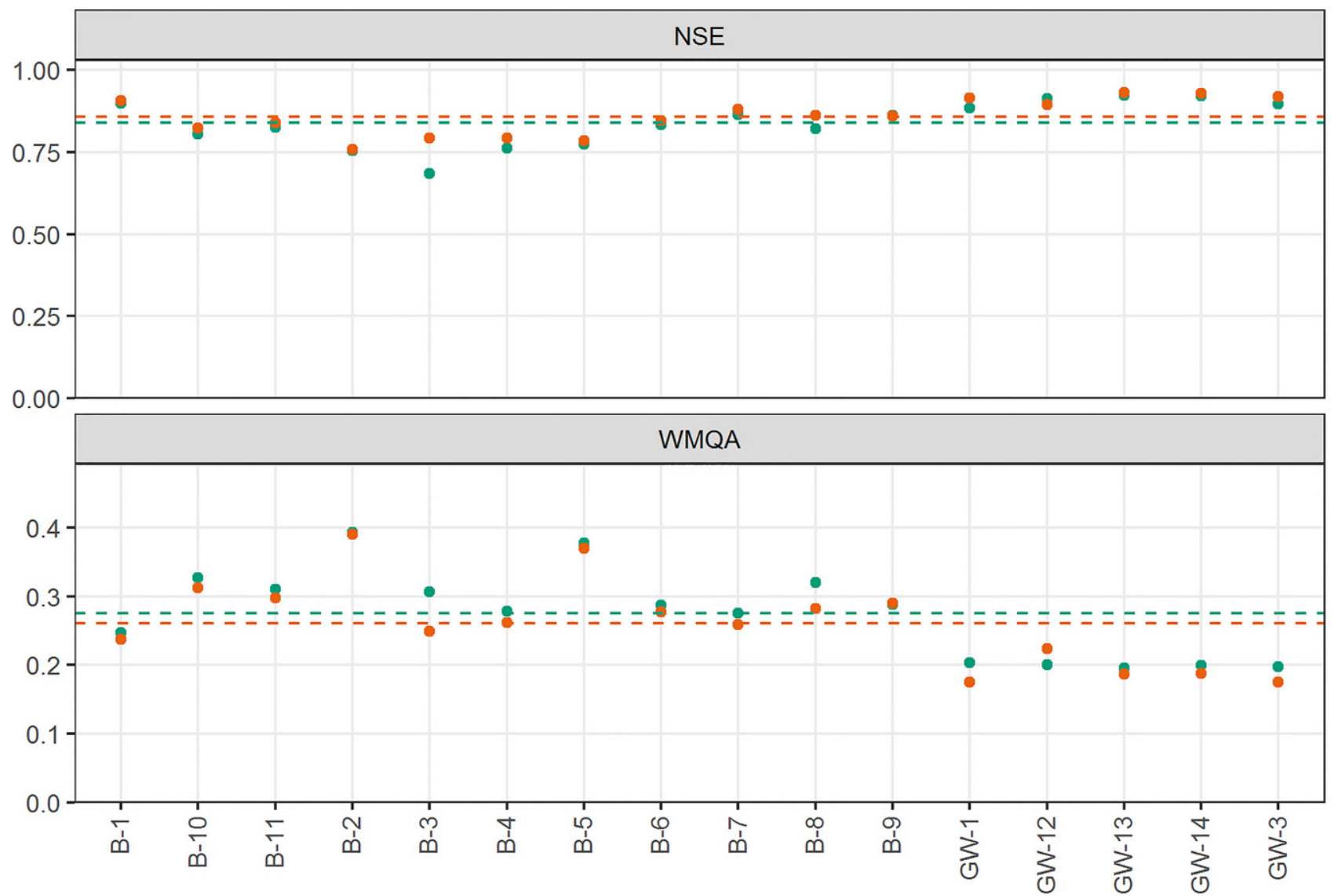

Kalibrierungsvariante 1 Kalibrierungsvariante 2

Abb. 9 Vergleich der Kalibrierungsvarianten anhand der Gütemaße 
Tab. 2 Mittlere Gütemaße der Kalibrierungsvarianten für alle Beobachtungsmessstellen

\begin{tabular}{l|l|l} 
Gütemaß & Kalibrierungsvariante 1 & Kalibrierungsvariante 2 \\
\hline WMQA $[\mathrm{cm}]$ & 28 & 26 \\
\hline NSE $[-]$ & 0,84 & 0,86
\end{tabular}

nie der Abflusskurven) einerseits durch eine Erhöhung des Zuflusses über die Gewässersohle (blaue Linie der Zuflusskurve) und einen verringerten Abfluss aus dem Modell über die Randzellen mit variablem Wasserstand (magentafarbene Linie in der Zuflusskurve) kompensiert.

Die grüne Linie stellt jene Wassermengen dar, die durch Änderungen des Wasserstands gebunden (Abflusskurve - Aufspiegelung durch Verringerung der Entnahme aus den Brunnen) und frei werden (Zuflusskurve - Absenkung durch Erhöhung der Entnahme aus den Brunnen).

Alle übrigen Randbedingungen weisen grundsätzlich einen ähnlichen Verlauf im Vergleich zwischen den beiden Varianten auf. Dies ist sehr gut in Abb. 7 erkennbar, in der die relativen Anteile der $\mathrm{Zu}$ - und Abflussraten über die einzelnen Modellgrenzen als relativer Anteil am Gesamtdurchsatz des jeweiligen Modells dargestellt sind.

Güte der Modellanpassung Die Parametersätze aus beiden Kalibrierungsvarianten wurden herangezogen und der gesamte Untersuchungszeitraum modelliert. Durch den Vergleich von simulierten Wasserständen aus dem Modell mit gemessenen Wasserständen in den Beobachtungsmessstellen wird die Güte der Modellanpassung der beiden Varianten dargestellt. Abb. 8 zeigt den direkten grafischen Vergleich in Form eines Streudiagramms. Dafür wurden die Werte-Paare von Beobachtung und Simulation $\mathrm{zu}$ den unterschiedlichen Zeitpunkten direkt gegeneinander aufgetragen. Bei perfekter Übereinstimmung liegen die Punkte auf der $45^{\circ}$ Geraden (rote Linie). Die beiden strichlierten roten Linien markieren Abweichungen von $50 \mathrm{~cm}$. Brunnen werden als „+“, Grundwassermessstellen als blaue Punkte dargestellt. In der Kalibrierungsvariante 2 liegen die Punkte etwas dichter um die $45^{\circ}$-Gerade. Dies weist auf eine geringfügig bessere Übereinstimmung zwischen Beobachtungen und Simulation in dieser Variante hin. Die größten Abweichungen werden in Brunnen gemessen. Dies ist unter anderem auch auf den steilen Absenktrichter zurückzuführen, der sich bei Vertikalfil- terbrunnen um den Brunnen ausbildet, wodurch der Wasserstand gerade in Brunnen, aus denen Wasser entnommen wird, etwas ungenauer modelliert werden kann.

Es werden zwei verschiedene Gütemaße für jede der 16 Beobachtungsmessstellen gesondert berechnet.

Die Wurzel der mittleren quadratischen Abweichung (WMQA - Gl. 2) gibt den gemittelten absoluten Fehler zwischen gemessenen und berechneten

Der Nash-Sutcliffe-Koeffizient (NSE - Gl. 3) ist ein Maß für die Aussagekraft eines Modells und gibt an, wie gut die Vorhersagekraft des Modells im Vergleich zum Mittelwert der gemessenen Größe ist. Er kann zwischen $-\infty$ und 1 liegen. Negative Werte zeigen an, dass der Mittelwert einer Messgröße genauer ist als die Vorhersage des Modells. Ein NSE von 0 bedeutet, dass die Vorhersagekraft des Modells jener des Mittelwerts der Messwerte entspricht. Bei einem NSE von 1 stimmen Beobachtung und Simulation perfekt überein. In der Hydrologie wird häufig ab NSE-Werten von 0,85 eine gute Anpassung des Modells an die Wirklichkeit attestiert.

$$
W M Q A=
$$

$$
\sqrt{\frac{1}{n_{\text {beob }}} \sum_{i=1}^{n_{\text {beob }}}\left(W S T_{i, \text { beob }}-W S T_{i, \text { sim }}\right)^{2}}
$$

MOQ Mittlere quadratische Abweichung [m]

$n_{\text {beob }} \quad$ Anzahl der Beobachtun$W_{S T} T_{i, b e o b}$ ben $\begin{array}{ll}W S T_{i, b e o b} & \text { beobachteter Wasser- } \\ \text { stand zum Zeitpunkt i }\end{array}$ $\begin{array}{ll}W_{\text {WST }} & {[\mathrm{m} \text { ü. A.] }} \\ & \text { simulierter Wasserstand }\end{array}$ zum Zeitpunkt i [m ü. A.]

$N S E=1-\frac{\sum_{1}^{n_{\text {beob }}}\left\{W S T_{i, \text { beob }}-W S T_{i, \text { sim }}\right\}^{2}}{\sum_{1}^{n_{\text {beob }}\left\{W S T_{i, \text { beob }}-W S T_{M W, \text { beob }}\right\}^{2}}}$

NSE Nash-Sutcliffe-Koeffizient [-] gen Messwerten in Metern an.

$n_{\text {beob }} \quad$ Anzahl der Beobachtun-
WST $T_{i, b e o b}$ beobachteter Wasserstand zum Zeitpunkt i [m ü. A.]

WST $_{i, \text { sim }} \quad$ simulierter Wasserstand zum Zeitpunkt i [m ü. A.]

$W S T_{M W, b e o b}$ Mittelwert aller beobachteten Wasserstände [m ü. A.]

Abb. 9 zeigt die Gegenüberstellung der Gütemaße beider Kalibrierungsvarianten der 16 Beobachtungsmessstellen. Die strichlierten Linien geben den Mittelwert der Gütemaße für die jeweilige Kalibrierungsvariante wieder. Diese werden in Tab. 2 angeführt. Die mittleren WMQAs von 26 bzw. $28 \mathrm{~cm}$ und mittleren NSE-Werte von 0,86 bzw. 0,84 drücken für beiden Parametersätzen eine sehr hohe Güte aus.

\section{Anwendung und Ergebnisse}

Mit den Parametersätzen aus beiden Kalibrierungsvarianten wurde jeweils der gesamte Untersuchungszeitraum modelliert. Aufgrund von Beschränkungen der Software in Bezug auf die Anzahl der festzulegenden Beobachtungen wurde der gesamte Untersuchungszeitraum dabei jeweils in 3 Abschnitte aufgeteilt. Die Randbedingungen, also die Wasserstände in den Randzellen und im Oberflächengewässer sowie die Entnahme aus den Brunnen, wurden entsprechend der Messungen eingegeben. Die zeitliche Diskretisierung wurde dabei so gewählt, dass zu jedem Probenahmezeitpunkt ein Zeitschritt endet. Bei der Simulation durch das Modell werden Differenzialgleichungen der Strömungsgleichung gelöst und dadurch die Vektoren der Fließgeschwindigkeit für jeden Zeitschritt in jeder Zelle der Modelle berechnet. Man erhält also das Strömungsmodell.

Zur Berechnung der Aufenthaltszeiten $\mathrm{zu}$ jedem Probenahmezeitpunkt wurde das Paket PM-Path verwendet, welches in die Modellierungssoftware integriert ist. Es baut auf die Ergebnisse des Strömungsmodells auf. Damit kann der advektive Stofftransport (Dispersion bleibt unberücksichtigt) berechnet werden. $\mathrm{Zu}$ jedem Probenahmezeitpunkt werden um die Zelle, welche den jeweiligen Entnahmepunkt repräsentiert, 1200 virtuelle Partikel positioniert. Danach wird in der Zeit rückwärts bis 100 Tage vor der Probenahme gerechnet und dabei die Bewegung aller Partikel verfolgt, bis sie entweder den Modell- 


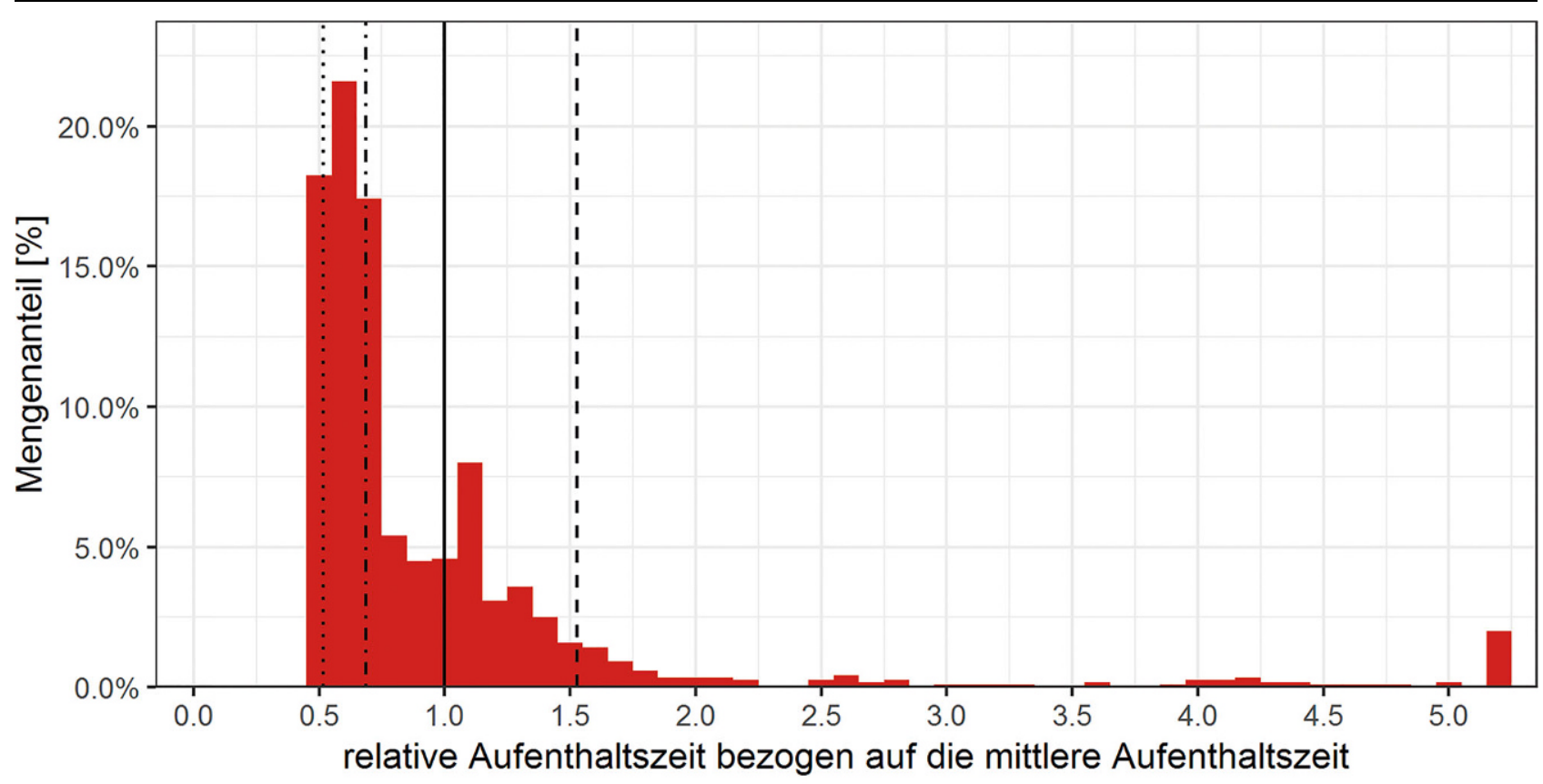

\section{Quantile $\vdots$ 10\%-Quantil $\vdots$ Median $\quad$ Mittelwert $\quad 90 \%$-Quantil}

Abb. 10 Beispiel der Verteilung der Aufenthaltszeit für einen Probenahmezeitpunkt

rand (z. B. Sohle des Oberflächengewässers) erreichen oder die maximale Simulationszeit von 100 Tagen erreicht ist.

Wasser, das zu einem bestimmten Zeitpunkt aus einem Brunnen entnommen wird, stellt immer eine Mischung aus Wässern dar, die über einen längeren Zeitraum hinweg in den Grundwasserkörper infiltriert sind. Die Aufenthaltszeit einer bestimmten Probe weist also eine Verteilung auf. Durch die Berechnung des Fließwegs für jedes Partikel kann eine Verteilung der Aufenthaltszeit für den jeweiligen Probenahmezeitpunkt geschätzt werden. Abb. 10 zeigt beispielhaft die Verteilung der Aufenthaltszeit für einen Probenahmezeitpunkt als Histogramm. Die Aufenthaltszeit wird relativ in Bezug auf den Mittelwert der Aufenthalts- zeiten (vertikale kontinuierliche Linie) dargestellt.

Die roten Balken stellen den Anteil an allen 1200 Partikeln dar, welche die jeweilige relative Aufenthaltszeit aufweisen. Die vertikalen Linien zeigen die Quantile der Verteilung. Der Median (strichpunktierte Linie) liegt hierbei knapp unter der 0,7-fachen mittleren Aufenthaltszeit. $10 \%$ der Partikel (punktierte Linie) haben eine halb so lange Aufenthaltszeit wie im Mittel. 90\% aller Partikel haben eine Aufenthaltszeit, die unter der 1,5-fachen mittleren Aufenthaltszeit liegt (90-\%-Quantil - strichlierte Linie).

Mithilfe der Erkenntnisse aus der Grundwassermodellierung kann eine deutlich verbesserte Aussage bei der Gegenüberstellung von Ergebnissen aus Wasserqualitätsanalysen aus dem
Fließgewässer mit dem Grund- bzw. Brunnenwasser getroffen werden.

Funding Open access funding provided by University of Natural Resources and Life Sciences Vienna (BOKU).

Open Access Dieser Artikel wird unter der Creative Commons Namensnennung 4.0 International Lizenz (http:// creativecommons.org/licenses/by/4. $0 /$ deed.de) veröffentlicht, welche die Nutzung, Vervielfältigung, Bearbeitung, Verbreitung und Wiedergabe in jeglichem Medium und Format erlaubt, sofern Sie den/die ursprünglichen $\mathrm{Au}$ tor(en) und die Quelle ordnungsgemäß nennen, einen Link zur Creative Commons Lizenz beifügen und angeben, ob Änderungen vorgenommen wurden.

\section{Literatur}

Chiang, W. H. and Kinzelbach, W (2001): 3DGroundwater Modeling with PMWIN. First Edition. Springer Berlin Heidelberg New York. ISBN 3-540 67744-5, 346 pp.

R Core Team (2018): R A Language and Environment for Statistical Computing. R Foundation for Statistical Computing, Vienna, Austria. URL: https://ww.R-project.org/

Hinweis des Verlags Der Verlag bleibt in Hinblick auf geografische Zuordnun- gen und Gebietsbezeichnungen in veröffentlichten Karten und Institutsadressen neutral. 